\title{
Identification of targets of miRNA-221 and miRNA-222 in fulvestrant-resistant breast cancer
}

\author{
PENGFEI LIU ${ }^{1,2^{*}}$, MANNA SUN ${ }^{3 *}$, WENHUA JIANG ${ }^{4}$, \\ JINKUN ZHAO $^{5}$, CHUNYONG LIANG $^{3}$ and HUILAI ZHANG ${ }^{1,2}$ \\ ${ }^{1}$ Department of Lymphoma, Tianjin Medical University Cancer Institute and Hospital, \\ National Clinical Research Center for Cancer, Key Laboratory of Cancer Prevention and Therapy; \\ ${ }^{2}$ Tianjin's Clinical Research Center for Cancer, Sino-US Center of Lymphoma and Leukemia, Tianjin 300060; \\ ${ }^{3}$ School of Material Science and Engineering, Hebei University of Technology, Tianjin 300322, \\ ${ }^{4}$ Department of Radiotherapy, The Second Hospital of Tianjin Medical University, Tianjin 300211; \\ ${ }^{5}$ Department of Radiology, Tianjin Medical University Cancer Institute and Hospital, \\ National Clinical Research Center for Cancer, Key Laboratory of Cancer Prevention and Therapy, Tianjin 300060, P.R. China
}

Received May 19, 2015; Accepted August 10, 2016

DOI: $10.3892 / \mathrm{ol} .2016 .5180$

\begin{abstract}
The present study aimed to identify the differentially expressed genes (DEGs) regulated by microRNA (miRNA)-221 and miRNA-222 that are associated with the resistance of breast cancer to fulvestrant. The GSE19777 transcription profile was downloaded from the Gene Expression Omnibus database, and includes data from three samples of antisense miRNA-221-transfected fulvestrant-resistant MCF7-FR breast cancer cells, three samples of antisense miRNA-222-transfected fulvestrant-resistant MCF7-FR cells and three samples of control inhibitor (green fluorescent protein)-treated fulvestrant-resistant MCF7-FR cells. The linear models for microarray data package in $\mathrm{R} /$ Bioconductor was employed to screen for DEGs in the miRNA-transfected cells, and the pheatmap package in $\mathrm{R}$ was used to perform two-way clustering. Pathway enrichment was conducted using the Gene Set Enrichment Analysis tool. Furthermore, a miRNA-messenger (m) RNA regulatory network depicting interactions between miRNA-targeted upregulated DEGs was constructed and visualized using Cytoscape. In total, 492 and 404 DEGs were identified for the antisense miRNA-221-transfected MCF7-FR cells and the antisense miRNA-222-transfected MCF7-FR cells, respectively. Genes of the pentose
\end{abstract}

Correspondence to: Dr Huilai Zhang, Department of Lymphoma, Tianjin Medical University Cancer Institute and Hospital, National Clinical Research Center for Cancer, Key Laboratory of Cancer Prevention and Therapy, 24 Huan-Hu-Xi Road, He Xi, Tianjin 300060, P.R. China

E-mail: snowlpf@163.com

${ }^{*}$ Contributed equally

Key words: breast cancer, microRNA, microarray, fulvestrant resistance, gene set enrichment analysis phosphate pathway (PPP) were significantly enriched in the antisense miRNA-221-transfected MCF7-FR cells. In addition, components of the Wnt signaling pathway and cell adhesion molecules (CAMs) were significantly enriched in the antisense miRNA-222-transfected MCF7-FR cells. In the miRNA-mRNA regulatory network, miRNA-222 was demonstrated to target protocadherin 10 (PCDH10). The results of the present study suggested that the PPP and Wnt signaling pathways, as well as CAMs and PCDH1O, may be associated with the resistance of breast cancer to fulvestrant.

\section{Introduction}

Breast cancer, which accounts for $\sim 23 \%$ of all newly diagnosed cases of cancer and was responsible for $14 \%(458,400)$ of all mortalities due to cancer in 2008, is the leading cause of cancer-associated mortality among females (1). A previous study reported that the estrogen receptor (ER), which is expressed in $\sim 75 \%$ of breast tumors, is considered the main target for the treatment of breast cancer, and women with breast tumors typically receive endocrine therapy (2).

Fulvestrant, which is a pure, steroidal antiestrogen, has been reported to completely suppress ER $\alpha$ activity by inactivating ER $\alpha$-mediated genomic and non-genomic signaling; it is considered a promising drug for the treatment of breast cancer in postmenopausal women (3). However, ER-targeted therapies fail in $\leq 50 \%$ of patients with breast tumors due to the occurrence of de novo or acquired resistance $(2,4)$. It has been reported that microRNAs (miRNAs) have a pivotal role in breast cancer, and the overexpression of miRNA-221/222 has been suggested to be associated with the emergence of fulvestrant resistance in breast cancer (5).

In 2011, Rao et al (6) used a microarray expression profile to identify differentially expressed genes (DEGs) between antisense miRNA-221-transfected or miRNA-222-transfected MCF7-FR cells and negative control-transfected MCF7-FR 
Table I. Top ten upregulated and downregulated DEGs in the antisense miRNA-221-transfected and antisense miRNA-222-transfected MCF7-FR cells, as compared with negative control-transfected MCF7-FR cells.

\begin{tabular}{|c|c|c|c|c|c|c|}
\hline \multirow[b]{2}{*}{ DEGs } & \multicolumn{3}{|c|}{ Antisense miRNA-221 vs. control } & \multicolumn{3}{|c|}{ Antisense miRNA-222 vs. control } \\
\hline & Gene & $\mid \log _{2} \mathrm{FCl}$ & adj.P.Val & Gene & $\mid \log _{2} \mathrm{FCl}$ & adj.P.Val \\
\hline \multirow[t]{10}{*}{ Downregulated } & LHX8 & -4.17614 & 0.000175 & $P T H$ & -3.49280 & 0.000184 \\
\hline & PSMB8 & -4.06074 & 0.000204 & PWAR5 & -3.27792 & 0.009442 \\
\hline & $F L G 2$ & -3.99026 & 0.001523 & $I Y D$ & -3.20234 & 0.001261 \\
\hline & TRPC5 & -3.83132 & 0.002291 & ICAM5 & -2.87824 & 0.017322 \\
\hline & OLFM4 & -3.51933 & 0.000522 & $D A O A-A S 1$ & -2.83576 & 0.044589 \\
\hline & KERA & -3.37161 & 0.006165 & STARD13-AS & -2.79578 & 0.005935 \\
\hline & GIMAP2 & -3.19819 & 0.008755 & WDR86-AS1 & -2.75129 & 0.021120 \\
\hline & CYP4F3OP & -2.92305 & 0.042475 & IZUMO2 & -2.68134 & 0.028787 \\
\hline & LOC100505635 & -2.89457 & 0.041871 & $R A G 2$ & -2.67795 & 0.006909 \\
\hline & $S L C 15 A 3$ & -2.88269 & 0.000007 & Clorf192 & -2.66616 & 0.022020 \\
\hline \multirow[t]{10}{*}{ Upregulated } & PRPS1L1 & 3.81365 & 0.002733 & OR2L13 & 3.55457 & 0.007431 \\
\hline & ARHGAP36 & 3.33814 & 0.000913 & PRPS1L1 & 3.54601 & 0.002105 \\
\hline & CXorf58 & 3.28644 & 0.000074 & SH3RF3-AS1 & 3.34868 & 0.000066 \\
\hline & LINC00567 & 3.24582 & 0.000653 & CXorf58 & 3.15695 & 0.028128 \\
\hline & $D Y D C 1$ & 3.18358 & 0.026382 & LOC 100505676 & 3.11364 & 0.001263 \\
\hline & OR2L13 & 2.88576 & 0.003734 & LINC00950 & 2.92266 & 0.001549 \\
\hline & $M L I P$ & 2.83880 & 0.036347 & NXPH1 & 2.90566 & 0.000380 \\
\hline & $K L K B 1$ & 2.79668 & 0.015902 & MSTN & 2.89563 & 0.000007 \\
\hline & LINC00950 & 2.75805 & 0.001693 & $D Z I P 1$ & 2.86233 & 0.007720 \\
\hline & OR10A5 & 2.74849 & 0.011940 & $C P E B 2-A S 1$ & 2.85775 & 0.015210 \\
\hline
\end{tabular}

FC, fold change; adj.P.Val, adjusted P-value; miRNA, microRNA; DEGs, differentially expressed genes.

cells, according to the cut-off criteria of $\mathrm{P}<0.05$ and $\log _{2}$ fold change $(\mathrm{FC}) \mid>1.2$. It was demonstrated that activation of $\beta$-catenin by miRNA-221/222 led to estrogen-independent growth and fulvestrant resistance, as well as to repression of transforming growth factor- $\beta$-mediated growth inhibition (6). However, another study reported different mechanisms for the occurrence of fulvestrant resistance in breast cancer (7). Tangkeangsirisin and Serrero (8) demonstrated that progranulin induced human breast cancer resistance to fulvestrant by inhibiting the apoptosis of breast cancer cells. In addition, the broad-spectrum metalloproteinase inhibitor BB-94 has been demonstrated to inhibit the growth of fulvestrant-resistant breast cancer cell lines, as well as the activation of human epidermal growth factor receptor 3 and extracellular signal-regulated kinase in these cells (9). Therefore, it is important to further screen for biomarkers associated with fulvestrant-resistance in breast cancer.

Using the same microarray data as Rao et al (6), the present study aimed to further screen for DEGs in antisense miRNA-221-transfected and antisense miRNA-222-transfected MCF7-FR cells. The linear models for microarray data (limma) package, based on a wide threshold range $(\mathrm{P}<0.05$ and $\log _{2} \mathrm{FCl}>1$ ), was used to identify DEGs associated with fulvestrant-resistant breast cancer. In addition, a Kyoto Encyclopedia of Genes and Genomes (KEGG) pathway enrichment analysis was performed, and the targets of miRNA-221/222 were predicted using miRanda and TargetScan. A previous study suggested that analyses based on different statistical tests may produce different outcomes (10). Therefore, the present study may obtain a number of results different from the data obtained in the initial study by Rao et al (6).

\section{Materials and methods}

Microarray data. The GSE19777 transcription profile used by Rao et al (6) was downloaded from the Gene Expression Omnibus database (http://www.ncbi.nlm.nih.gov/geo/). The profile was based on the GPL570 dataset, which was obtained using the [HG-U133_Plus_2] Affymetrix Human Genome U133 Plus 2.0 Array (Affymetrix, Inc., Santa Clara, CA, USA). In total, nine samples were included in the dataset, including three samples of antisense miRNA-221-transfected fulvestrant-resistant MCF7-FR breast cancer cells, three samples of antisense miRNA-222-transfected fulvestrant-resistant MCF7-FR cells and three samples of control inhibitor (green fluorescent protein)-treated fulvestrant-resistant MCF7-FR cells (negative control). In addition, the probe annotation information mapping the probes of genes was downloaded from Bioconductor (http:// www.bioconductor.org/).

Dataset preprocessing and DEG analysis. The $\mathrm{R}$ package from Affymetrix, Inc., was used to normalize the raw CEL data from the DNA microarrays (11). The downloaded expression profile was mapped to the corresponding 
A

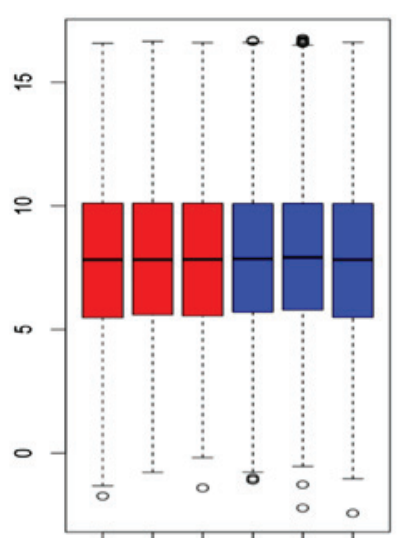

B

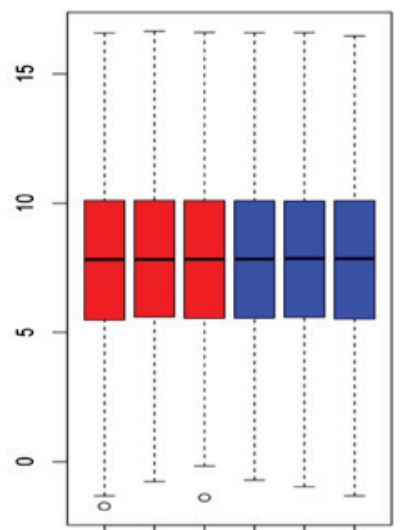

C

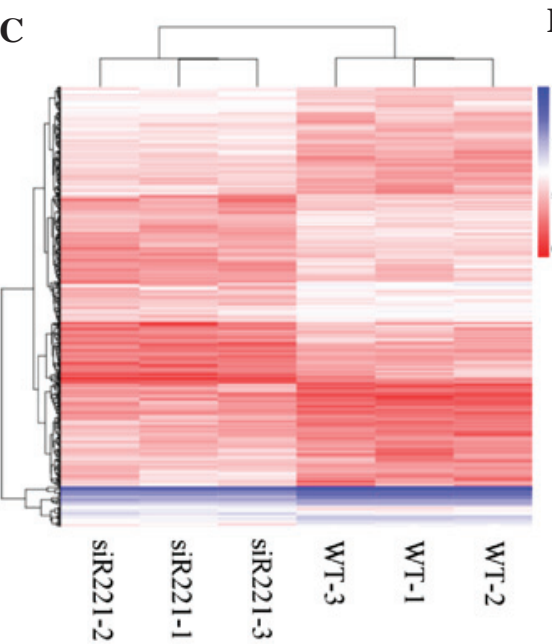

D

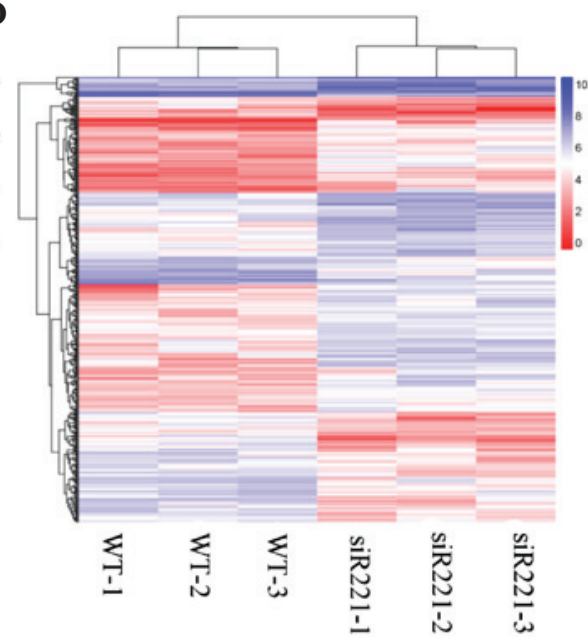

Figure 1. Box plots for the normalized gene expression data and the hierarchical clusters of DEGs. (A) Box plots for the normalized gene expression data of the antisense miRNA-221-transfected MCF7-FR breast cancer cells (red box plots) and the negative control-transfected MCF7-FR cells (blue box plots). (B) Box plots for the normalized gene expression data of the antisense miRNA-222-transfected MCF7-FR cells (red box plots) and the negative control-transfected MCF7-FR cells (blue box plots). (C) Hierarchical cluster analysis of DEGs in the antisense miRNA-221-transfected MCF7-FR cells, as compared with the negative control-transfected MCF7-FR cells. (D) Hierarchical cluster analysis of DEGs in the antisense miRNA-222-transfected MCF7-FR cells, as compared with the negative control-transfected MCF7-FR cells. The horizontal axis represents the samples. The vertical axis represents the DEGs. The color key indicates the expression value of the DEG. siR221, antisense miRNA-221-transfected MCF7-FR cells; DEGs, differentially expressed genes; miRNA, microRNA.

gene symbols. Average expression values were used for the genes with multiple probes. Subsequently, the limma package in R/Bioconductor (https://bioconductor. org/packages/release/bioc/html/limma.html) was used to screen for DEGs in the antisense miRNA-221-transfected and miRNA-222-transfected MCF7-FR cells, as compared with the negative control. The cut-off criteria for the DEGs were $\mathrm{P}<0.05$ and $\log _{2} \mathrm{FCl}>1$. The top ten upregulated and downregulated genes in the antisense miRNA-221-transfected and antisense miRNA-222-transfected MCF7-FR cells are indicated in Table I. Next, the pheatmap package (https:// cran.r-project.org/web/packages/pheatmap/index.html) in $\mathrm{R}$ was used to perform two-way clustering (12), based on the Euclidean distance (13).

Gene set enrichment analysis (GSEA). GSEA, which is a computational method that determines whether an a priori defined set of genes exhibits statistically significant and concordant differences between two biological states (14), was used to conduct the pathway enrichment analysis based on the expression levels of DEGs in the antisense miRNA-221-transfected and miRNA-222-transfected MCF7-FR cells. A gene count between 15 and 500 and $\mathrm{P}<0.01$ were set as the criteria to filter the pre-defined gene sets. In addition, the distant regulatory elements of co-regulated genes tool (http://dire. dcode.org), which enables the prediction of distant regulatory elements in higher eukaryotic genomes (15), was applied to screen for transcription factors associated with the DEGs in the enriched pathways.

miRNA-messenger (m) RNA regulatory network construction. Prediction of the targets of miRNA-221 and miRNA-222 was performed using the miRanda algorithm (http://microrna. sanger.ac.uk/targets/v5/) and TargetScan 4.2 (http://www. targetscan.org/). Subsequently, the miRNA-mRNA regulatory network, depicting interactions between the miRNAs and target DEGs (upregulated DEGs only) was constructed and visualized using Cytoscape (16).

\section{Results}

Preprocessing and DEG analysis. The box plots of the expression values for all genes in every sample following normalization are represented in Fig. 1A and B. In total, 


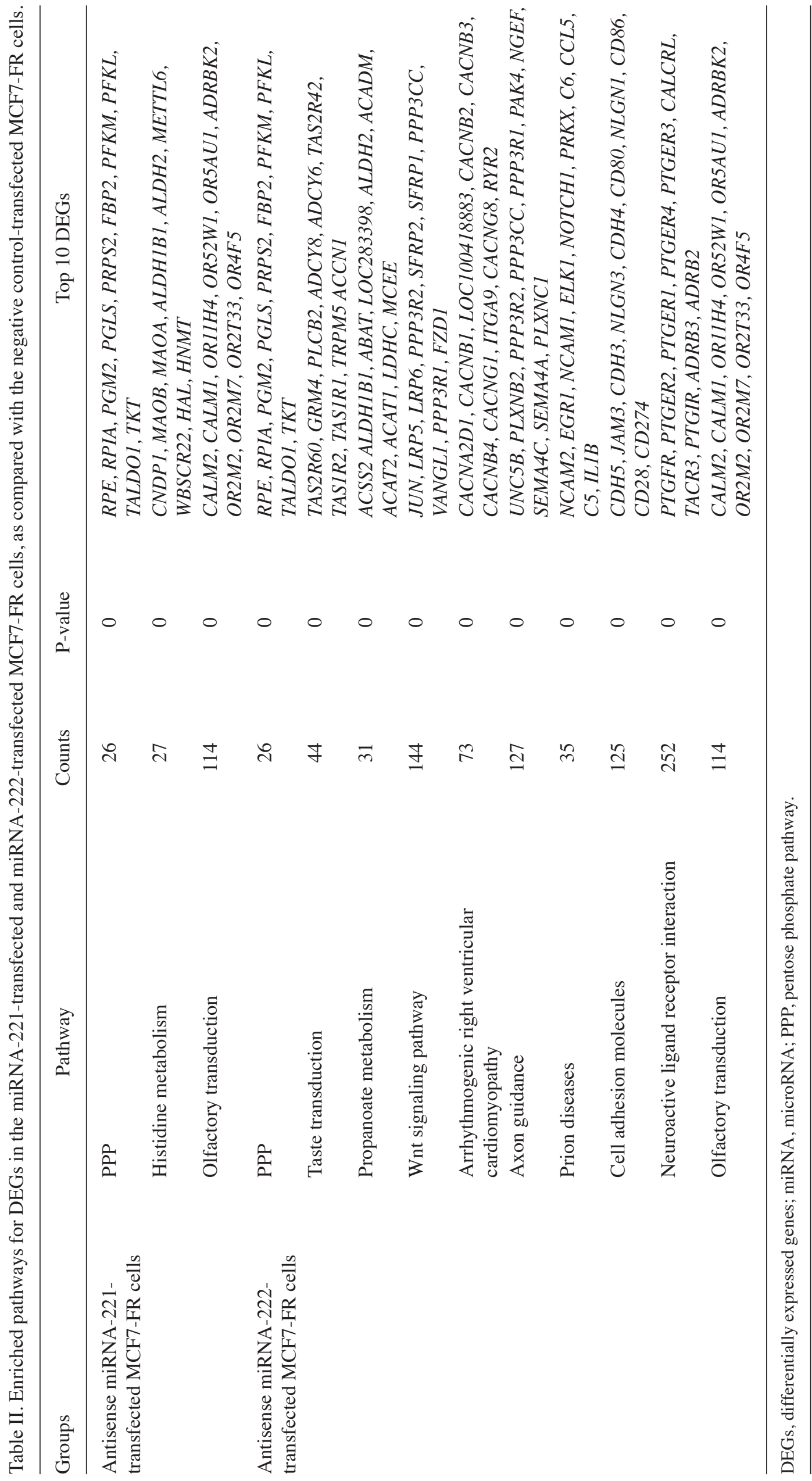


Table III. Counts of transcription factors for the differentially expressed genes in the enriched pathways.

\begin{tabular}{|c|c|c|}
\hline Group & KEGG pathway & Count \\
\hline \multirow[t]{3}{*}{ Antisense miRNA-221-transfected MCF7-FR cells } & PPP & 87 \\
\hline & Histidine metabolism & 80 \\
\hline & Olfactory transduction & 95 \\
\hline \multirow[t]{10}{*}{ Antisense miRNA-222-transfected MCF7-FR cells } & PPP & 87 \\
\hline & Taste transduction & 76 \\
\hline & Propanoate metabolism & 76 \\
\hline & Wnt signaling pathway & 94 \\
\hline & Arrhythmogenic right ventricular cardiomyopathy & 116 \\
\hline & Axon guidance & 104 \\
\hline & Prion diseases & 79 \\
\hline & Cell adhesion molecules & 123 \\
\hline & Neuroactive ligand receptor interaction & 113 \\
\hline & Olfactory transduction & 95 \\
\hline
\end{tabular}

KEGG, Kyoto Encyclopedia of Genes and Genomes; miRNA, microRNA; PPP, pentose phosphate pathway.

492 DEGs, including 247 upregulated [such as phosphoribosyl pyrophosphate synthetase 1-like 1 (PRPS1L1) and secreted frizzled-related protein 5 (SFRP5)] and 245 downregulated (such as LIM homeobox 8 and proteasome subunit beta 8 ) DEGs, were identified in the antisense miRNA-221-transfected MCF7-FR cells compared with the negative control, while 404 DEGs, including 255 upregulated [such as PRPS1L1 and claudin 8 (CLDN8)] and 149 downregulated (such as parathyroid hormone and Prader Willi/Angelman region RNA 5) DEGs, were identified in the antisense miRNA-222-transfected MCF7-FR cells compared with the negative control. The two-way hierarchical cluster analyses of the DEGs in the miRNA-221- and miRNA-222-transfected cells are represented in Fig. $1 C$ and D.

GSEA. Three pathways were significantly enriched in the antisense miRNA-221-transfected MCF7-FR cells compared with the negative control, while ten pathways were significantly enriched in the miRNA-221-transfected MCF7-FR cells compared with the negative control (Table II). In addition, two pathways, including the pentose phosphate pathway (PPP) and olfactory transduction, were enriched in both the antisense miRNA-221-transfected and miRNA-222-transfected MCF7-FR cells, as compared with the negative control (Table II). Notably, the DEGs SFRP5 and CLDN8 were significantly enriched in the Wnt signaling pathway and the cell adhesion molecules (CAMs) pathway, respectively (Table II).

Screening for transcription factors associated with the genes in the enriched pathways identified 123 transcription factors associated with the genes in the CAMs pathway. Furthermore, 94 transcription factors were associated with the genes enriched in the Wnt signaling pathway, and 87 transcription factors were associated with the genes enriched in the PPP (Table III).

Regulatory network analysis. According to the TargetScan and miRanda databases, 530 genes were targets of miRNA-221

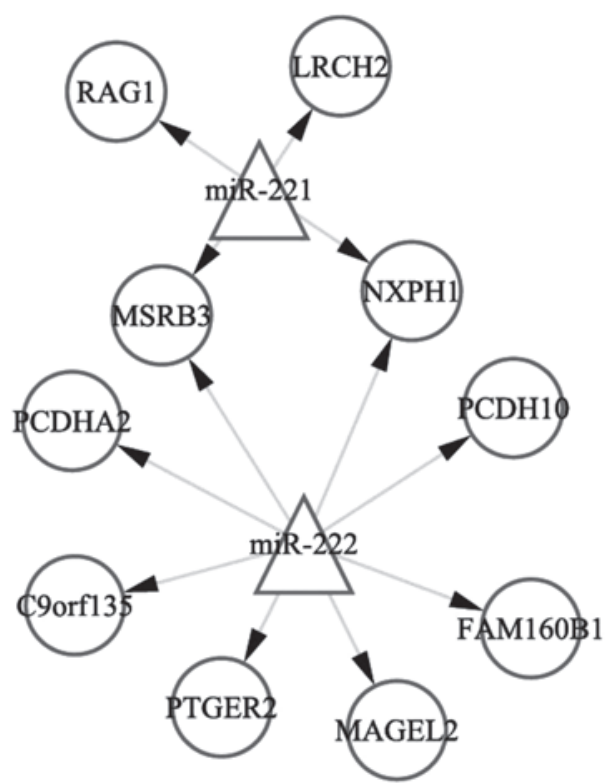

Figure 2. miRNA (miRNA-221 and miRNA-222)-target regulatory network. Triangles represent the miRNAs; circles represent the differentially expressed genes in miRNA-221- and miRNA-222-transfected MCF7-FR breast cancer cells. miRNA, microRNA; RAG1, recombination-activating gene 1; LRCH2, leucine-rich repeats and calponin homology domain containing 2; MSRB3, methionine sulfoxide reductase B3; NXPH1, neurexophilin 1; PCDHA2, protocadherin $\alpha-2$; PCDH10, protocadherin-10; C9orf135, chromosome 9 open reading frame 135; PTGER2, prostaglandin E receptor 2; MAGEL2, MAGE family member L2; FAM160B1, family with sequence similarity 160 member B1.

and 488 genes were targets of miRNA-221. Of the 530 target genes of miRNA-221, six were DEGs, including four upregulated genes [recombination activating gene 1, leucinerich repeats and calponin homology domain containing 2, methionine sulfoxide reductase B3 (MSRB3) and neurexophilin $1(N X P H 1)]$, in the antisense miRNA-221-transfected MCF7-FR cells. Of the 488 target genes of miRNA-222, ten 
were DEGs, including eight upregulated genes (MSRB3, $\mathrm{NXPH1}$, protocadherin $(\mathrm{PCDH}) \mathrm{A} 2, \mathrm{PCDH} 10$, chromosome 9 open reading frame 135, prostaglandin $\mathrm{E}$ receptor 2, MAGElike-2 and family with sequence similarity 160 member B1), in the antisense miRNA-222-transfected MCF7-FR cells. The miRNA-target regulatory network is represented in Fig. 2.

\section{Discussion}

In the present study, 492 and 404 DEGs were identified in the antisense miRNA-221-transfected MCF7-FR cells and the antisense miRNA-222-transfected MCF7-FR cells, respectively, as compared with the negative control. GSEA revealed that the PPP was significantly enriched in the antisense miRNA-221-transfected and antisense miRNA-222-transfected MCF7-FR cells. Furthermore, 87 transcription factors were identified for the genes enriched in the PPP, which suggested that the PPP was significantly regulated in these cells. The PPP produces two substrates, ribose 5-phosphate and nicotinamide adenine dinucleotide phosphate, which are necessary for the division of cells and serve as buffers to prevent reactive oxygen species-induced cell death and apoptosis (17). Alterations in the PPP activity have been reported to occur during cancer development and progression (18). In addition, an increase in the levels of various PPP metabolites in the breast epithelia, including sedoheptulose 7-phosphate and hexose phosphate intermediates, has been reported to occur during the transition from normal breast epithelial cells to transformed cells, as well as during the transition from non-metastatic to metastatic tumors $(19,20)$.

In the present study, the Wnt signaling pathway was significantly enriched in the antisense miRNA-222-transfected MCF7-FR cells compared with the normal control-transfected cells. A total of 94 transcription factors were associated with the genes enriched in the Wnt signaling pathway, which suggested that this pathway was highly regulated in the miRNA-222-transfected MCF7-FR cells. A previous study reported that the activation of the Wnt signaling pathway could lead to the metastasis of breast cancer (21). In addition, the blockage of Wnt signaling has been demonstrated to inhibit cell proliferation and migration, and to induce apoptosis in triple-negative breast cancer cells (22).

In the present study, the CAMs pathway was significantly enriched in the antisense miRNA-222-transfected MCF7-FR cells compared with the normal control-transfected cells. A total of 123 transcription factors were associated with the genes enriched in this pathway. CAMs are membrane receptors that mediate cell-cell and cell-matrix interactions, and have an essential role in transducing intracellular signals responsible for adhesion, migration, invasion, angiogenesis and organ-specific metastasis (23). Adhesion molecules, including E-cadherin and carcinoembryonic antigen, have been associated with the process of metastasis in breast cancer cells (24). Taken together, these results suggested that the PPP, Wnt signaling pathway and CAMs pathway may be associated with the resistance of breast cancer to fulvestrant.

In the miRNA-target regulatory network, miR-222 was observed to target PCDH1O. PCDH1O is a member of the mammalian cadherin superfamily, which has key roles in cell migration and calcium-dependent, cadherin-mediated homophilic cell-cell interactions (25). A previous study identified $P C D H 1 O$ as a candidate tumor suppressor in nasopharyngeal, esophageal and various other carcinomas, in which it was associated with frequent methylation (26). As a result, $P C D H 10$ targeted by miR-222 could be associated with the resistance of breast cancer to fulvestrant.

In conclusion, the results of the present study suggested that the PPP, Wnt signaling pathway and CAMs KEGG pathway, as well as $P C D H 10$, may be associated with the development of fulvestrant resistance in patients with breast cancer. However, further studies are required to elucidate the underlying mechanisms.

\section{Acknowledgements}

The present study was supported by the Health Bureau Science and Technology Foundation of Tianjin (Tianjin, China; grant no. 2012KZ063) and the National Natural Science Foundation of China (Beijing, China; grant nos. 81302082, 81272685, 31301151 and 81172355 ).

\section{References}

1. Jemal A, Bray F, Center MM, Ferlay J, Ward E and Forman D: Global cancer statistics. CA Cancer J Clin 61: 69-90, 2011.

2. Ariazi EA, Ariazi JL, Cordera F and Jordan VC: Estrogen receptors as therapeutic targets in breast cancer. Curr Top Med Chem 6: 181-202, 2006

3. Howell A: Fulvestrant ('Faslodex'): Current and future role in breast cancer management. Crit Rev Oncol Hematol 57: 265-273, 2006.

4. Baumgarten SC and Frasor J: Minireview: Inflammation: An instigator of more aggressive estrogen receptor (ER) positive breast cancers. Mol Endocrinol 26: 360-371, 2012.

5. Xin F,Li M, Balch C, Thomson M, Fan MY,Liu Y,Hammond SM, Kim S and Nephew KP: Computational analysis of microRNA profiles and their target genes suggests significant involvement in breast cancer antiestrogen resistance. Bioinformatics 25: 430-434, 2009

6. Rao X, Di Leva G, Li M, Fang F, Devlin C, Hartman-Frey C, Burow ME, Lvan $M$, Croce $C M$ and Nephew KP: MicroRNA-221/222 confers breast cancer fulvestrant resistance by regulating multiple signaling pathways. Oncogene 30: 1082-1097, 2011.

7. Perey L, Paridaens R, Hawle H, Zaman K, Nolé F, Wildiers H, Fiche M, Dietrich D, Clément P, Köberle D, et al: Clinical benefit of fulvestrant in postmenopausal women with advanced breast cancer and primary or acquired resistance to aromatase inhibitors: Final results of phase II Swiss Group for Clinical Cancer Research Trial (SAKK 21/00). Ann Oncol 18: 64-69, 2007.

8. Tangkeangsirisin W and Serrero G: GP88 (Progranulin) confers fulvestrant (Faslodex, ICI 182,780) resistance to human breast cancer cells. Adv Breast Cancer Res 3: 68-78, 2014.

9. Kirkegaard T, Yde CW, Kveiborg M and Lykkesfeldt AE: The broad-spectrum metalloproteinase inhibitor BB-94 inhibits growth, HER3 and Erk activation in fulvestrant-resistant breast cancer cell lines. Int J Oncol 45: 393-400, 2014.

10. Afsari B, Geman D and Fertig EJ: Learning dysregulated pathways in cancers from differential variability analysis. Cancer Inform 13 (Suppl 5): S61-S67, 2014.

11. Troyanskaya O, Cantor M, Sherlock G, Brown P, Hastie T, Tibshirani R, Botstein D and Altman RB: Missing value estimation methods for DNA microarrays. Bioinformatics 17: 520-525, 2001.

12. Székely GJ and Rizzo ML: Hierarchical clustering via joint between-within distances: Extending Ward's minimum variance method. J Classif 22: 151-183, 2005.

13. Deza E and Deza MM (eds): Encyclopedia of distances. 3rd edition. Springer-Verlag, Berlin, 2009.

14. Shi J and Walker MG: Gene Set Enrichment Analysis (GSEA) for Interpreting Gene Expression Profiles. Curr Bioinform 2: 133-137, 2007. 
15. Gotea V and Ovcharenko I: DiRE: Identifying distant regulatory elements of co-expressed genes. Nucleic Acids Res 36 (Web Server Issue): W133-W139, 2008.

16. Smoot ME, Ono K, Ruscheinski J, Wang PL and Ldeker T: Cytoscape 2.8: New features for data integration and network visualization. Bioinformatics 27: 431-432, 2011.

17. Tian WN, Braunstein LD, Apse K, Pang J, Rose M, Tian X and Stanton RC: Importance of glucose-6-phosphate dehydrogenase activity in cell death. Am J Physiol 276: C1121-C1131, 1999.

18. Riganti C, Gazzano E, Polimeni M, Aldieri E and Ghigo D: The pentose phosphate pathway: An antioxidant defense and a crossroad in tumor cell fate. Free Radic Biol Med 53: 421-436, 2012.

19. Richardson AD, Yang C, Osterman A and Smith JW: Central carbon metabolism in the progression of mammary carcinoma. Breast Cancer Res Treat 110: 297-307, 2008.

20. Lu X, Bennet B, Mu E, Rabinowitz J and Kang Y: Metabolomic changes accompanying transformation and acquisition of metastatic potential in a syngeneic mouse mammary tumor model. J Biol Chem 285: 9317-9321, 2010.
21. Cai J, Guan H, Fang L, Yang Y, Zhu X, Yuan J, Wu J and Li M: MicroRNA-374a activates Wnt/ $\beta$-catenin signaling to promote breast cancer metastasis. J Clin Invest 123: 566-579, 2013.

22. Bilir B, Kucuk O and Moreno CS: Wnt signaling blockage inhibits cell proliferation and migration, and induces apoptosis in triple-negative breast cancer cells. J Transl Med 11: 280. 2013.

23. Li DM and Feng YM: Signaling mechanism of cell adhesion molecules in breast cancer metastasis: Potential therapeutic targets. Breast Cancer Res Treat 128: 7-21, 2011.

24. Saadatmand S, de Kruijf EM, Sajet A, Dekker-Ensink NG, van Nes JG, Putter H, Smit VT, van de Velde CJ, Liefers GJ and Kuppen JK: Expression of cell adhesion molecules and prognosis in breast cancer. Br J Surg 100: 252-260, 2013.

25. Pines J, Toldo L and Lafont F: Cell to cell contact and extracellular matrix. Curr Opin Cell Biol 10: 561, 1998.

26. Ying J, Li H, Seng TJ, Langford C, Srivastava G, Tsao SW, Putti T, Murray P, Chan AT and Tao Q: Functional epigenetics identifies a protocadherin PCDH10 as a candidate tumor suppressor for nasopharyngeal, esophageal and multiple other carcinomas with frequent methylation. Oncogene 25: 1070-1080, 2006. 